\title{
A preliminary study into the sensitivity of disease activity detection by serial weekly magnetic resonance imaging in multiple sclerosis
}

\author{
M Lai, T Hodgson, M Gawne-Cain, S Webb, D MacManus, W I McDonald, \\ A J Thompson, D H Miller
}

\begin{abstract}
Long TR and gadolinium enhanced spin echo brain MRI was performed weekly for three months in three patients with relapsing-remitting or secondary progressive multiple sclerosis. During the study, 38 new enhancing lesions were seen; 11 showed enhancement for less than four weeks, and two enhanced on only one scan. All 16 new lesions seen on long TR scans showed initial enhancement. When only every fourth (monthly) scan was analysed, a total of 33 new enhancing lesions were seen. Subject to confirmation in a larger cohort, the results suggest: (a) that blood brain barrier leakage is an invariable event in new lesion development in relapsing-remitting and secondary progressive multiple sclerosis; $(b)$ the small increase in sensitivity of weekly scanning does not justify its use in preference to monthly scanning when monitoring treatments.
\end{abstract}

(F Neurol Neurosurg Psychiatry 1996;60:339-341)

Keywords: magnetic resonance imaging; multiple sclerosis

Serial brain MRI at monthly intervals has provided valuable insights into the natural history of multiple sclerosis, and is now often used to monitor the efficacy of experimental treatments. ${ }^{12}$ Scanning at this interval often shows asymptomatic new lesions in relapsing-remitting or secondary progressive multiple sclerosis, on average five to 10 times more often than clinical relapse. ${ }^{3-10}$ About $80 \%$ of new lesions on unenhanced conventional long TR spin echo scans show gadolinium enhancement (on T1 weighted spin echo sequences), ${ }^{5611}$ indicating a breach of the blood-brain barrier. Gadolinium enhancement correlates with pathological features of activity-namely, perivascular lymphocyte and macrophage infiltration and myelin breakdown, ${ }^{3} 1213$ and has usually ceased at the next monthly follow up although the associated long TR abnormality persists. Foci of enhancement are also often found in the absence of a definite corresponding change on the long TR images. ${ }^{11}$ Thus the power of MRI in monitoring the effect of lesion activity is increased by acquiring enhanced images in addition to standard long TR scans.

Two important questions arising are: (1) does increasing the frequency of MRI detect more active lesions, thus improving the sensitivity of the technique for therapeutic monitoring; (2) do new lesions appearing on monthly long TR scans without showing gadolinium enhancement display enhancement at shorter intervals-evidence that they do would suggest that impairment of the blood-brain barrier is an obligatory early event in the development of lesions in multiple sclerosis. To consider these questions, we have performed weekly MRI for three months in three patients, one with relapsing-remitting and two with secondary progressive multiple sclerosis.

\section{Patients and methods}

PATIENT SELECTION

The patients chosen had to have clinically definite multiple sclerosis ${ }^{14}$ of the relapsing-remitting or secondary progressive type ${ }^{56}$ and had to have gadolinium enhancement on their first scan. These criteria were to increase the possibility of a high yield of new gadolinium enhancing lesions during the study. ${ }^{11}$ Of 11 patients screened only three had enhancing lesions. Thus eight patients did not continue after their first scan.

\section{MRI PROTOCOL}

Informed consent for gadolinium enhanced MRI was obtained from each patient before the commencement of the study.

Each patient was given $0.1 \mathrm{mmol} / \mathrm{kg}$ gadolinium-DTPA intravenously. The MRI protocol was then performed as follows:

Firstly, three sets of pilot scans were performed for the purposes of repositioning. These were a T1 weighted sagittal scan, an axial scan, and a coronal scan. After this an axial fast spin echo sequence was used to acquire long TR scans through the cerebral hemispheres $(T R=1500 \mathrm{~ms}$, TEf $=32 / 80$ $\mathrm{ms}$, slice number 16 , slice thickness $5 \mathrm{~mm}$, field of view $24 \mathrm{~cm}$, matrix $256 \times 128$ ). Gadolinium enhanced $T 1$ weighted images were then obtained $(T R=500 \mathrm{~ms}, \mathrm{TE}=14$ ms, slice number 16 , slice thickness $5 \mathrm{~mm}$, field of view $24 \mathrm{~cm}$, matrix $256 \times 128$ ). 
Top row: $T 1$ weighted gadolinium enhanced MRIs. Bottom row: equivalent long TR scan The sequences show the development of two lesions $(A$ and $B)$. Lesion $A$ enhances for one scan only with changes on the long $T R$ scan one week later. Lesion $B$ continued to enhance on six scans. $A$ new lesion appeared on the long TR scan simultaneously with the enhancement. acquired in the same study.

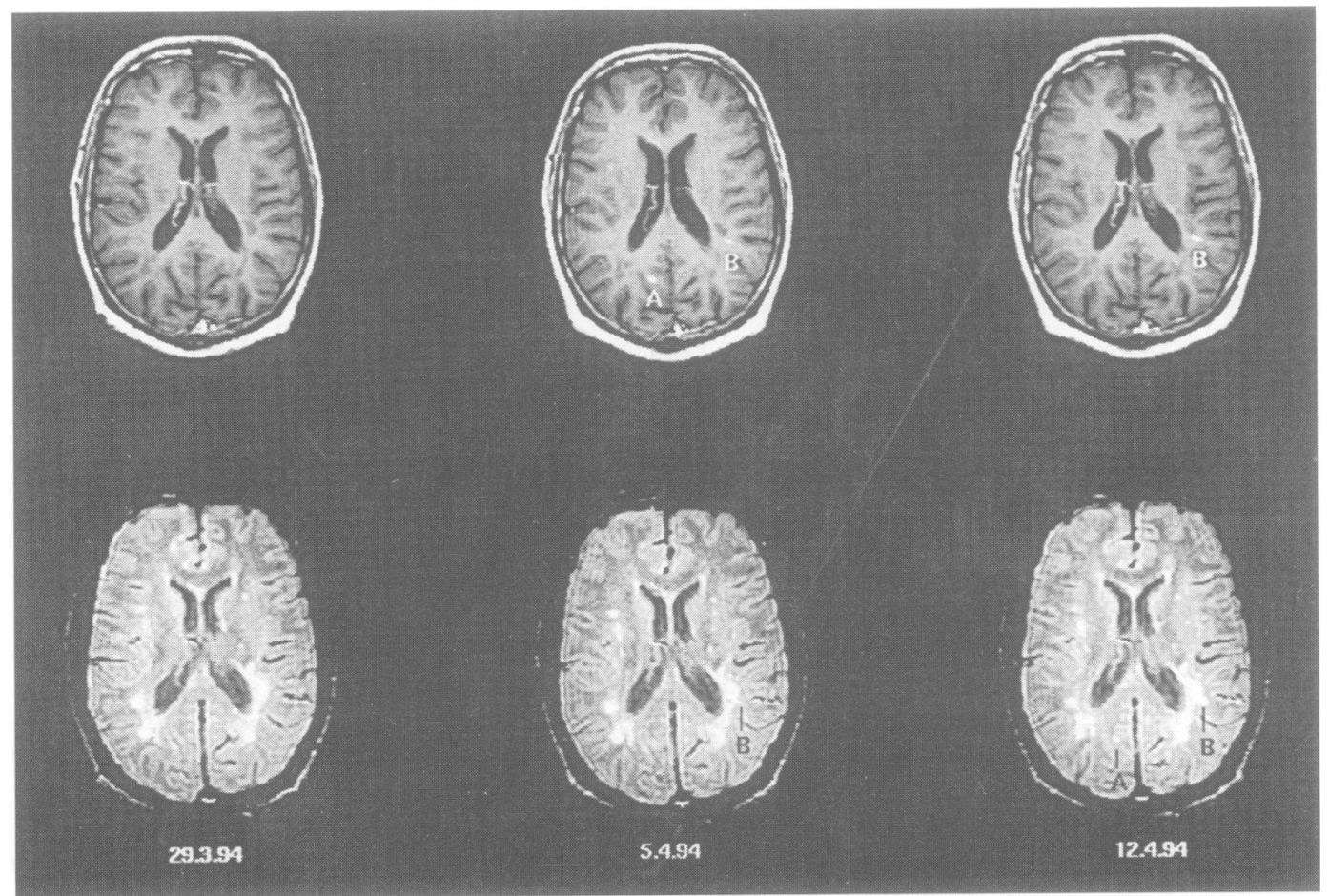

Thirteen weekly studies were performed with the above protocol on each patient. Lesion activity was reported from hard copies by two radiologists (TH, MG-C).

\section{Results}

CLINICAL DATA

Patient 1 (aged 38 years) had relapsing-remitting multiple sclerosis. At the start of the study her expanded disability status scale (EDSS) ${ }^{15}$ score was 6 . She had one mild relapse in the fourth week of the study. At the end of the study her EDSS score was still 6 .

Patient 2 (aged 35 years) had secondary progressive multiple sclerosis. The initial

Duration of enhancement of the 25 lesions in which enhancement could be followed from the beginning to the end

\begin{tabular}{|c|c|c|c|c|c|c|c|c|c|c|c|c|c|}
\hline \multirow[b]{2}{*}{ Lesion number } & \multicolumn{13}{|c|}{ Week of study } \\
\hline & 1 & 2 & 3 & 4 & 5 & 6 & 7 & 8 & 9 & 10 & 11 & 12 & 13 \\
\hline 1 & & $x$ & $x$ & $x$ & $x$ & $x$ & $x$ & & & & & & \\
\hline 2 & & & & $x$ & $x$ & $x$ & $x$ & $x$ & & & & & \\
\hline 3 & & & & $x$ & $x$ & $x$ & $x$ & $x$ & & & & & \\
\hline 4 & & & & $x$ & $x$ & $x$ & & & & & & & \\
\hline 5 & & & & $x$ & $x$ & $x$ & $x$ & $x$ & & & & & \\
\hline 6 & & & & & & $x$ & $x$ & $x$ & & & & & \\
\hline 7 & & & & & & $x$ & $x$ & $x$ & $x$ & $x$ & $x$ & & \\
\hline 8 & & & & & 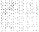 & $x$ & $x$ & $x$ & $x$ & $x$ & $x$ & $x$ & \\
\hline 9 & & & & & & & & & $x$ & $x$ & $x$ & & \\
\hline 10 & & & & $x$ & $x$ & $x$ & $x$ & $x$ & $x$ & $x$ & & & \\
\hline 11 & & & & & $x$ & $x$ & $x$ & $x$ & $x$ & $x$ & & & \\
\hline 12 & & & & & & $x$ & $x$ & $x$ & & & & & \\
\hline 13 & & & & & & & $x$ & $x$ & $x$ & $x$ & $x$ & & \\
\hline 14 & & & & & & & $x$ & $x$ & & & & & \\
\hline 15 & & & & & & & & & $x$ & $\times$ & & & \\
\hline 16 & & $x$ & $x$ & $x$ & $x$ & & & & & & & & \\
\hline 17 & & $x$ & $x$ & $x$ & $x$ & $x$ & & & & & & & \\
\hline 18 & & & $x$ & $x$ & $x$ & $x$ & $x$ & $x$ & $x$ & $x$ & $x$ & $x$ & \\
\hline 19 & & & & $x$ & $x$ & $x$ & $x$ & $x$ & $x$ & $x$ & & & \\
\hline 20 & & & & & $x$ & & & & & & & & \\
\hline 21 & & & & & $x$ & $x$ & $\times$ & & & & $x$ & & \\
\hline $\begin{array}{l}22 \\
23\end{array}$ & - & & & & & & & $\begin{array}{l}x \\
x\end{array}$ & $\hat{x}$ & $\hat{x}$ & ^ & & \\
\hline 24 & & & & & & & & $x$ & & & & & \\
\hline 25 & & & & & & & & & & $x$ & $x$ & $x$ & \\
\hline
\end{tabular}

Weeks $1,5,9$, and 13 are shaded and simulate the analysis points of the "monthly" scans. An " $x$ " indicates the presence of enhancement of a lesion. Thus at the monthly time points the 24 could be clearly seen as new lesions on the long TR scan.
EDSS was 6. Further decline was observed during the study and the final EDSS was 7. progressive multiple sclerosis. The initial EDSS was 7. She had no further decline during the study.

MRI DATA

The weekly gadolinium enhanced scans were reviewed first. During follow up, 38 new enhancing lesions appeared. Thirteen were still enhancing on the final scan. Of these 13, enhancement was present on six scans in one lesion, five scans in two, four scans in three, three scans in one, two scans in three, and the final scan only in three.

Of the 25 new enhancing lesions which could be followed up from the beginning to the end of enhancement, $11(44 \%)$ enhanced for less than one month (on three scans or less). Two lesions enhanced on just one scan (figure).

To compare the sensitivity of weekly and monthly scanning, the first, fifth, ninth, and 13th scans were considered to be the monthly scans for this study. These scans showed 33 new enhancing lesions. Thus monthly scanning would have missed only five of $38(13 \%)$ new enhancing lesions detected on weekly scans (see table: lesions $6,12,14,24$, and 25).

The weekly proton density and T2 weighted (long TR) scans were then reviewed without reference to the enhanced images. A total of 16 new lesions was seen. The images were then compared with the weekly enhanced scans, and it was apparent that all the new lesions on long TR scans displayed gadolinium enhancement at their first appearance. However, two of the new lesions on long TR were no longer enhancing on the next "monthly" scan. Thus monthly scanning showed 35 new lesions, 33 enhancing and two not (see table: lesions 12 and 24), whereas
Patient 3 (aged 40 years) had secondary 
weekly scanning showed 38 new lesions, all enhancing.

\section{Discussion}

This study of three patients with relapsingremitting (one patient) or secondary progressive (two patients) multiple sclerosis was intended to compare the sensitivity of weekly versus monthly brain $M R I$ in detecting active lesions in multiple sclerosis, and to elucidate the consistency with which leakage of the blood-brain barrier occurs in the initial stages of new lesion formation. We are aware of only one other study in which weekly gadolinium enhanced scanning was performed, but in this the sensitivity of weekly versus monthly scans and the duration of enhancement were not described. ${ }^{16}$

As in a previous study of serial monthly scans, ${ }^{11}$ we found that gadolinium enhancement markedly increased the yield of newly active lesions compared with unenhanced long TR SE images alone. The reasons for the greater sensitivity of enhanced scans have been discussed in detail elsewhere. ${ }^{11}$ However, there was only a slight drop in the number of enhancing lesions comparing monthly with weekly enhanced scans. The slightly lower sensitivity of monthly scans should not appreciably alter the power of MRI to monitor disease activity in natural history or treatment trial studies, and if confirmed in a further study of a larger cohort, it would seem that the major additional burden to the patient and cost of weekly examinations will not be justified in such a setting.

On weekly scanning, every new lesion on long TR images showed an initial phase of gadolinium enhancement. The study establishes that enhancement sometimes lasts for less than two weeks, and it seems likely that new non-enhancing long TR lesions seen on monthly scans do have a brief phase of enhancement (as indeed seen in two lesions in the present study). Although we have only studied three patients, the consistency of this finding suggests that breakdown of the bloodbrain barrier is an invariable and perhaps obligatory event in the development of new lesions in relapsing-remitting or secondary progressive multiple sclerosis. Such a conclusion supports earlier work in which enhancement has been found to precede the appearance of the long TR lesions in four instances, and clinical relapse in one. ${ }^{17}$

Unlike the relapsing forms of the disease, gadolinium enhancement is infrequently seen in new long TR lesions on monthly scans of patients with primary progressive multiple sclerosis, a relatively uncommon subgroup of patients in whom there is progressive deterioration in symptoms from onset without relapses. ${ }^{5}$ We wondered whether such lesions might enhance but for a shorter duration than those in patients with relapsing disease, and we performed weekly scans in four primary progressive patients to explore this possibility (unpublished data). There were, however, no new lesions during that study, reflecting the low frequency with which they appear in this group.
An alternative explanation is that there is a low grade leak to the blood-brain barrier which is not detectable using standard doses of contrast. Pathologically, low grade inflammation is indeed seen in primary progressive multiple sclerosis $^{17}$ and a recent study using a higher dose of contrast agent $(0.3 \mathrm{mmol} / \mathrm{kg} \mathrm{Gd}-$ DTPA) has disclosed a substantial increase in the number of enhancing lesions. ${ }^{19}$

The role of the blood-brain barrier in the development of new lesions and its relation to the patient's clinical state are important issues which need to be considered. In this regard techniques are needed which maximise the sensitivity of MRI to impairment of the bloodbrain barrier. These include the use of higher doses of gadolinium chelates, magnetisation transfer $\mathrm{T} 1$ weighted sequences, ${ }^{20}$ and $3 \mathrm{D}$ volume $\mathrm{T} 1$ weighted gradient echo sequences.

1 Miller DH, Barkhof F, Berry I, Kappos L, Scotti G, Thompson AJ. Magnetic resonance imaging in monitoring the treatment of multiple sclerosis: concerted action guidelines. $\mathcal{F}$ Neurol Neurosurg Psychiatry 1991;54:683-8.

2 McFarland HF, Frank JA, Albert PS, et al. Using gadolinium enhanced magnetic resonance imaging lesions to monitor disease activity in multiple sclerosis. Ann Neurol 1992;32:758-66.

3 Isaac $\mathrm{C}, \mathrm{Li} \mathrm{DKB}$, Genton $\mathrm{M}$, et al. Multiple sclerosis, a serial study using MRI in relapsing patients. Neurology 1988;38:1511-5.

4 Bastianello S, Pozzilli C, Bernardi S, et al. Serial study of gadolinium-DTPA MRI enhancement in multiple sclerogadolinium-DTPA MRI enha

5 Thompson AJ, Kermode AG, Wicks D, et al. Major differences in the dynamics of primary and secondary progressive multiple sclerosis. Ann Neurol 1991;29:53-62.

6 Thompson AJ, Miller DH, Youl B, et al. Serial gadolinium enhanced MRI in relapsing remitting multiple sclerosis of varying disease duration. Neurology 1992;42:60-3.

7 Harris JO, Frank JA, Patronas N, McFarlin DE, McFarland HF. Serial gadolinium enhanced magnetic resonance imaging scans in patients with early, relapsingand natural history. Ann Neurol 1991;29:548-55.

8 Capra R, Marciano N, Vignolo LA, Chiesa A, Gasporotti R. Gadolinium-pentetic acid magnetic resonance imaging in patients with relapsing remitting multiple sclerosis. Arch Neurol 1992;49:687-9.

9 Barkhof F, Scheltens P, Frequin STFM, et al. Relapsingremitting multiple sclerosis: sequential enhanced MR imaging vs clinical findings in determining disease activity. imaging vs clinical findings in determinin

10 Barkhof F, Valk J, Hommes OR, Scheltens P, Nauta JJP. Gadopentate dimeglumine enhancement of multiple sclerosis lesions on long TR spin-echo images at $0 \cdot 6 \mathrm{~T}$. AfNR Am $\mathcal{f}$ Neuroradiol 1992;13:1257-9.

11 Miller DH, Barkhof F, Nauta JJP. Gadolinium enhancement increases the sensitivity of MRI in detecting disease activity in multiple sclerosis. Brain 1993;116:1077-94.

12 Katz D, Taubenberger J, Raine C, McFarlin D, McFarland HF. Gadolinium enhancing lesions on magnetic resonance imaging: neuropathological findings. Ann Neurol 1990;28:243-6.

13 Rodriguez M, Scheithauer BW, Forbes G, Kelly PJ Oligodendrocyte injury is an early event in lesions of multiple sclerosis. Mayo Clin Proc 1993;68:627-36.

14 Poser CM, Paty DW, Scheinberg L, et al. New diagnostic criteria for multiple sclerosis: guidelines for research protocols. Ann Neurol 1983;13:227-31.

15 Kurtzke JF. Rating neurological impairment in multiple sclerosis: an expanded disability status scale (EDSS) Neurology 1983;33:1444-52.

16 Khoury SJ, Guttman CR, Orav EJ, et al. Longitudinal MRI in multiple sclerosis: correlation between disability and lesion burden. Neurology 1994;44: 2120-4.

17 Kermode BE, Thompson AJ, Kingsley DPE, Mosely IF, Rudge P, McDonald WI. Breakdown of the blood-brain barrier precedes other MRI signs of new lesions in multiple sclerosis: pathogenetic and clinical implications. Brain 1990;113:1477-89.

18 Revesz T, Kidd D, Thompson AJ, Barnard RO, McDonald WI. A comparison of the pathology of primary and secWI. A comparison of the pathology of primary and sec-
ondary progressive multiple sclerosis. Brain 1994;117: ondary prog $759-65$.

19 Fillipi M, Campi A, Martinelli V, Colombo B, Yousry T, Canal N, Scotti G, Comi G. Comparision of triple dose versus standard dose gadolinium-DTPA for detection of MRI enhancing lesions in patients with primary progressive multiple sclerosis. I Neurol Neurosurg Psychiatry 1995;59:540-4.

20 Finelli DA, Hurst GC, Gullapali RP, Bellon EM. Improved contrast of enhancing brain lesions on postgadolinium $\mathrm{T} 1$-weighted spin-echo images with use of magnetization transfer. Radiology 1992;190:553-9. 